\title{
Functional and laminar dissociations between muscarinic and nicotinic cholinergic neuromodulation in the tree shrew primary visual cortex
}

\author{
Anwesha Bhattacharyya, ${ }^{1,2}$ Felix Bießmann, ${ }^{1,3}$ Julia Veit, ${ }^{1,2}$ Robert Kretz ${ }^{1}$ and Gregor Rainer ${ }^{1,2}$ \\ ${ }^{1}$ Visual Cognition Laboratory, Department of Medicine, University of Fribourg, Chemin du Musée 5, CH-1700 Fribourg, Switzerland \\ ${ }^{2}$ Fribourg Center for Cognition, University of Fribourg, Fribourg, Switzerland \\ ${ }^{3}$ Machine Learning Group, Berlin Institute of Technology, Franklinstrasse 28/29, D-10587 Berlin, Germany
}

Keywords: Acetylcholine, contrast gain, neural computation, Nicotine, orientation tuning, Oxotremorine

\begin{abstract}
Acetylcholine is an important neuromodulator involved in cognitive function. The impact of cholinergic neuromodulation on computations within the cortical microcircuit is not well understood. Here we investigate the effects of layer-specific cholinergic drug application in the tree shrew primary visual cortex during visual stimulation with drifting grating stimuli of varying contrast and orientation. We describe differences between muscarinic and nicotinic cholinergic effects in terms of both the layer of cortex and the attribute of visual representation. Nicotinic receptor activation enhanced the contrast response in the granular input layer of the cortex, while tending to reduce neural selectivity for orientation across all cortical layers. Muscarinic activation modestly enhanced the contrast response across cortical layers, and tended to improve orientation tuning. This resulted in highest orientation selectivity in the supra- and infragranular layers, where orientation selectivity was already greatest in the absence of pharmacological stimulation. Our results indicate that laminar position plays a crucial part in functional consequences of cholinergic stimulation, consistent with the differential distribution of cholinergic receptors. Nicotinic receptors function to enhance sensory representations arriving in the cortex, whereas muscarinic receptors act to boost the cortical computation of orientation tuning. Our findings suggest close homology between cholinergic mechanisms in tree shrew and primate visual cortices.
\end{abstract}

\section{Introduction}

Cholinergic neuromodulation plays an important role in cognitive processes, including attention, learning and memory formation (Lucas-Meunier et al., 2003; Hasselmo \& Sarter, 2011). Cholinergic projections to the cortex originate in nuclei of the basal forebrain, and supply acetylcholine diffusely to the neocortical mantle (Mesulam et al., 1992). The cholinergic projections do have some degree of anatomical specificity, such that for example cholinergic levels in the primary visual cortex can be regulated independently of other cortical areas (Fournier et al., 2004; Laplante et al., 2005). Because acetylcholine is released mostly by volume transmission and not directly at synapses, its release has relatively low spatial specificity, and its actions are therefore largely determined by the location of cholinergic receptors. A lot is known about the distribution of the two cholinergic receptor types, the muscarinic (mAChR) and nicotinic (nAChR) acetylcholine receptors (Dani \& Bertrand, 2007; Brown, 2010), both regarding cortical layer and differential expression on excitatory or inhibitory neurons. For example, a high density of nAChR is present presynaptically on thalamo-cortical projection neurons in the granular

Correspondence: Dr G. Rainer, as above.

E-mail: gregor.rainer@unifr.ch input layer of cortex (Prusky et al., 1987; Disney et al., 2007), whereas $\mathrm{mAChRs}$ are more abundant in the superficial layers (Disney et al., 2006). Because of the differential distribution of receptors across the six layers of cortex, stimulating $\mathrm{nAChR}$ and $\mathrm{mAChR}$ with laminar specificity might offer the possibility to disentangle various effects associated with cholinergic neurotransmission. This approach could thus provide a clearer picture of cholinergic modulation in sensory cortex in comparison with previous findings that have often not systematically examined cholinergic responses by layer and receptor subtype, and have reported diverse and sometimes conflicting results (Sillito \& Kemp, 1983; Sato et al., 1987; Murphy \& Sillito, 1991; Zinke et al., 2006; Roberts \& Thiele, 2008).

Along the lines of laminar-specific electrophysiological recordings, activating the nAChRs present on thalamo-cortical synapses in the granular layer of macaque visual cortex (specifically layer IVc) strongly enhanced the response of V1 neurons to visual stimuli only in this layer in the macaque monkey, by increasing the response gain of the visual response (Disney et al., 2007). A recent study has partially confirmed this finding, but also revealed strong effects of $\mathrm{mAChR}$ activation on the V1 contrast response (Soma et al., 2011), particularly outside of the cortical input layer.

We take these results in macaques as a starting point for our study in tree shrews, by asking whether this response gain enhancement is also 
observed in this close relative of primates. We continue by addressing the question of how this gain in sensory input affects the cortical computation of orientation selectivity, the key attribute of V1 neurons, which is thought to arise by both selective combination of unoriented feed-forward input (Chapman \& Stryker, 1992) (Reid \& Alonso, 1996) as well as by cortico-cortical inhibitory interactions (Shapley et al., 2003). In addition, we investigate whether activation of $\mathrm{mAChR}$ also has any effects on the contrast response and orientation tuning, to provide a more unified understanding of cholinergic impact on sensory representations. Our work in tree shrew is of interest from a comparative point of view, and also because close homology of cholinergic processing to primates would contribute to further establishing the tree shrew as a valuable animal model for studying V1 circuitry and pharmacology.

\section{Materials and methods}

\section{Animal preparation}

Experiments were performed on eight adult tree shrews (Tupaia belangeri) aged 2-5 years. Animals were initially anesthetized with ketanarkon (i.m. $100 \mathrm{mg} / \mathrm{kg}$ ), and then Atropine (i.m. $0.02 \mathrm{mg} / \mathrm{kg}$ ) was used to reduce mucus secretion. The muscle relaxant pancuronium bromide was used (i.p., initial dose $0.4 \mathrm{mg} / \mathrm{kg}$, then $0.2 \mathrm{mg} / \mathrm{kg}$ approximately every $45 \mathrm{~min}$ ). Animals were artificially respirated at 100 strokes/min (Harvard Instruments Respirator) using a mixture of $70 \% \mathrm{~N}_{2} \mathrm{O}$ and $30 \% \mathrm{O}_{2}\left(95 \% \mathrm{O}_{2} / 5 \% \mathrm{CO}_{2}\right)$ and isoflurane $(0.5-1.5 \%)$ administered using a vaporizer (Dräger). The animal was then transferred to a stereotaxic device (David Kopf) that was modified to permit visual stimulation. Animals wore contact lenses to prevent drying of eyes. An eye drop of atropine was applied to the eye for pupil dilation. Visual stimulation was monocular, the other eye was covered. To gain access to the primary visual cortex, the temporal muscle was removed, the bone cleaned and a hole $(\sim 4 \mathrm{~mm}$ diameter $)$ was drilled around $\mathrm{AP}-1 \mathrm{~mm}$ and $\mathrm{ML}+4 \mathrm{~mm}$. A small slit was made in the dura using a syringe needle to permit introduction of the electrode pipette into the cortex. After electrode placement, the cortex was covered with lukewarm $2 \%$ agarose (in $0.9 \% \mathrm{NaCl}$ ) to prevent it from drying and provide stability. During the entire experiment, body temperature was maintained at $37{ }^{\circ} \mathrm{C}$ via an electric heating pad controlled by a rectal thermal probe (Harvard Instruments). All procedures were approved by local authorities and were in full compliance with applicable Swiss as well as European (European Communities Council Directive 86/609/EEC) animal experimentation guidelines.

\section{Electrophysiology and iontophoresis}

Recordings and drug injection were performed in the primary visual cortex with the help of a three-barreled borosilicate glass capillary (Thiele et al., 2006). The flanking capillaries had a different outer and inner diameter than the central capillary [flanking capillaries: outer diameter (o.d.) $0.545 \pm 0.1 \mathrm{~mm}$, inner diameter (i.d.) $0.273 \pm 0.1 \mathrm{~mm}$, filament $0.05 \mathrm{~mm}$; central capillary: o.d. $1.0 \pm 0.1 \mathrm{~mm}$, i.d. $0.6 \pm 0.1 \mathrm{~mm}$, no filament, length $120 \mathrm{~mm}$; Hilgenberg $\mathrm{GmBH}]$. There was no filament present in the central glass capillary where the tungsten electrode was inserted. For recording of extracellular action potentials local to the site of injection, a tungsten rod of $125 \mu \mathrm{m}$ (Advent Research Materials, UK) was inserted and glued in the central barrel after electrolytic etching, which resulted in a diameter of $1 \mu \mathrm{m}$ at the tip. The three-barrel micropipette was pulled with a Kopf pipette puller (Model 720) to obtain a total tip diameter of $10 \mu \mathrm{m}$. The sealed flanking barrels and the tungsten electrode tip that were still covered by glass were ground using a microgrinder with the aid of a microscope. The tungsten electrode had a final impedance of 1-2 M $\Omega$, and the barrels had a resistance of 15-80 M $\Omega$. In each experiment, one iontophoretic barrel was filled with solutions of either Nicotine hydrogen tartrate $(0.25 \mathrm{M}, \mathrm{pH} 3$; Sigma) or Oxotremorine, a muscarinic agonist ( $0.1 \mathrm{M}, \mathrm{pH} 5$; Sigma), dissolved in distilled water. An ejection current of (30-100 nA) was applied to a silver wire immersed in the solution for drug application using a Neurophore BH2 system (Harvard Instruments). The other barrel, which was filled with saline $(0.9 \%)$, was connected to the automatic current balance, which automatically compensated for charge delivery during iontophoretic drug application. The currents chosen for drug delivery depended on the resistance of the barrels, and were set at values low enough to prevent epileptiform bursting responses in the spontaneous activity in the absence of visual stimulation. We did not systematically vary the injection current, but used generally only one current per recording site, as dose-dependence was not of particular interest in the current study. A retention current of $-10 \mathrm{nA}$ was used to prevent any drug leakage during baseline and recovery periods.

The electrode pipette was advanced into the primary visual cortex using a hydraulic microdrive (David Kopf Instruments). For a given penetration, we recorded activity at multiple depths. The signal was amplified by a RA16PA Medusa preamplifier, and then filtered and digitized by a RZ5 Bioamp Processor (Tucker-Davis Technologies, Alachua, FL, USA). Action potential waveforms were recorded by thresholding the signal filtered between $300 \mathrm{~Hz}$ and $4 \mathrm{kHz}$, and sampled at $24.4 \mathrm{kHz}$. Although we took care to isolate single units, it is difficult to be sure in all cases that waveforms are truly due to single neuron activity when using single electrodes, as opposed for example to tetrode recordings where sorting into single units can be done with greater confidence. We thus designate our spiking activity to be of multi-unit origin.

\section{Visual stimulation}

Stimuli were generated with Psychophysics Toolbox running on a Mac Mini and presented on a gamma corrected 21" Compaq Qvision 210 cathode ray tube monitor running at $119.22(120 \mathrm{~Hz})$. Maximum luminance measured with a Minolta TV-color analyser was determined as $50 \mathrm{~cd} / \mathrm{m}^{2}$. Before recording, we mapped the receptive fields location by manually sliding bars generated with a simple graphics program back and forth on the monitor. The stimulus was then positioned in this area, centered at eccentricities between $8^{\circ}$ and $13^{\circ}$, and generally with a Gaussian aperture of radius $7.5^{\circ}$. Stimuli were drifting sine wave gratings of eight directions $\left(0^{\circ}, 45^{\circ}, 90^{\circ}, 135^{\circ}\right.$, $180^{\circ}, 225^{\circ}, 270^{\circ}, 315^{\circ}$ and $\left.360^{\circ}\right)$ and three contrasts $(10 \%, 50 \%$ and $100 \%$ ), masked with a Gaussian aperture, so called Gabor Wavelets. Spatial and temporal frequencies of the grating were manually adjusted to maximize the neural responses. Spatial frequencies were between 0.05 and 0.4 cycles/deg, and temporal frequencies were 2 or 3 cycles/s. The stimuli were presented for $2 \mathrm{~s}$ in pseudorandom order separated by inter-stimulus intervals of $1 \mathrm{~s}$.

\section{Data analysis}

We extracted multi-unit spiking activity and computed for each presented grating the mean spike frequency $R_{i}$. We generally used three repetitions of each unique stimulus condition (eight directions, three contrasts), because we wanted to limit the total duration per iontophoretic injection to no more than 4 min to prevent saturation 
effects, in line with previous studies (Zinke et al., 2006; Disney et al., 2007; Soma et al., 2011). For the contrast analyses, we always averaged data at each contrast across all directions, so that each data point represents a mean of $3 \times 8=24$ trials. For the contrast response curve, summing over the mean spike rate for each contrast yielded the area under the contrast response curve (CRFA). We fitted Naka-Rushton functions to the contrast response curve: $r(c)=R_{\max } \frac{c^{n}}{c^{n}+c_{50}^{n}}+R_{0}$, where the parameters peak firing rate $\left(R_{\max }\right)$, baseline firing rate $\left(R_{0}\right)$, semi-saturation contrast $\left(C_{50}\right)$ and an exponent parameter $(n)$ are obtained.

The preferred orientation of each site was determined by finding the stimulus (at $100 \%$ contrast) that maximized the expression $R_{\text {pref }}-\left(R_{\text {orth } 1}+R_{\text {orth } 2}\right) / 2$, where $R_{\text {pref }}$ is the preferred orientation and $R_{\text {orth }(i)}$ are the two orthogonal orientations. In order to quantify how well a given site is tuned to orientation, we computed an orientation tuning index: OTI $=\sqrt{\left(\sum\left(\mathrm{R}_{\mathrm{i}} \sin 2 \theta_{\mathrm{i}}\right)\right)^{2}+\left(\sum\left(R_{i} \cos 2 \theta_{i}\right)\right)^{2}} / \sum R_{i}$, where $R_{i}$ are the mean responses observed at orientations $\theta_{i}$. The $R_{i}$ values represent averages over $3 \times 2=6$ repetitions, corresponding to contrasts of $50 \%$ and $100 \%$ at each direction. The OTI values are thus based on a total of 48 trials. To determine orientation tuning parameters, we fitted wrapped normal distributions to the orientation tuning function: $r(\theta)=R_{0}+R_{\max } \sum_{-5}^{+5} e^{-(\theta+\mu-2 \pi k) / 2 \sigma^{2}}$, with parameters of preferred orientation $(\mu)$, baseline firing rate $\left(R_{0}\right)$, peak firing rate $\left(R_{\max }\right)$ and standard deviation $(\sigma)$. From these fits, we determined tuning height $\left(\mathrm{TH}=R_{\max }-R_{0}\right)$ and tuning width (TW $=\sigma$ ) parameters. Because we considered orientation and not direction in these fits, we thus averaged data from pairs of directions corresponding to the same orientation. The fits are thus based on $2 \times 2 \times 3=12$ repetitions for each orientation (considering again $50 \%$ and $100 \%$ contrast trials), yielding a total of 48 trials per Gaussian fit.

\section{Histology}

At the end of a recording session, two to four electrolytic lesions were made using a constant current stimulator (WPI A360) passing $10 \mu \mathrm{A}$ for $5 \mathrm{~s}$ at different depths through the tungsten electrode. Animals were then perfused through the heart with $0.9 \% \mathrm{NaCl}$ followed by icecold $4 \%$ paraformaldehyde in $0.1 \mathrm{M}$ phosphate buffer ( $\mathrm{pH} 7.4)$. The top of the skull was removed, and a coronal cut was made through the brain at AP $+4 \mathrm{~mm}$ in the stereotaxic frame. The brain was then removed and immersed in a mixture of $2 \%$ dimethylsulfoxide first in $10 \%$ and later $20 \%$ glycerol in $0.1 \mathrm{M}$ phosphate buffer $(\mathrm{pH}$ 7.4) until they sank. The posterior part of the brain was then cut sagitally into $50-\mu \mathrm{m}$ serial sections using a freezing microtome (Microm HM440E). The sections were treated with cytochrome C (Sigma) and diaminobenzidine (Sigma) for cytochrome oxidase immunohistochemistry (Wong-Riley, 1979); this facilitated visualization of laminar boundaries. The localization of the recording sites was then determined by reconstruction along the electrode tracks using lesion sites as reference marks, thereby assigning each recorded cell to a cortical layer.

\section{Results}

We recorded activity from 139 units in the primary visual cortex of eight tree shrews, using penetrations that were close to perpendicular to the cortical surface. Electrode position was verified by electrolytic lesions along the electrode track using cytochrome oxidase histochemistry (see Materials and methods; Fig. 1A). As visual stimuli we employed drifting gratings, using the preferred spatial (typically between 0.05 and 0.4 cycles/deg) and temporal frequency (typically

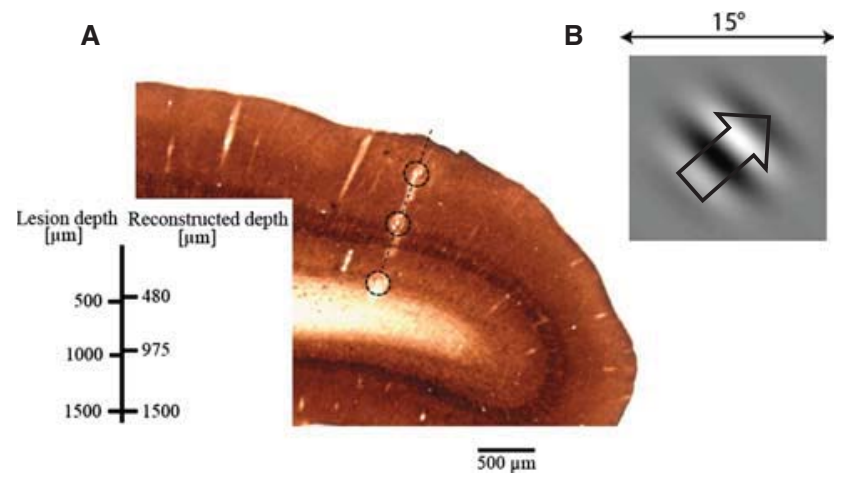

FIG. 1. Anatomical data and experimental setup. (A) Electrode position was verified histologically by making multiple lesions at different penetration depths (lesion depth) and comparing these to the position measurements made during data acquisition using a microdrive (reconstructed depth), yielding close correspondence between these values. (B) Drifting grating stimulus used to activate V1.

about 2 cycles/s) for each recording site, as determined by a manual mapping procedure (Fig. 1B). We used eight grating drift directions separated by $45^{\circ}$ and three contrast levels (10\%, 50\% and 100\%). The stimuli were presented for $2 \mathrm{~s}$ in pseudorandom order, separated by inter-stimulus intervals of $1 \mathrm{~s}$. Each unit was studied in three phases: a baseline phase prior to drug application; the drug application phase; and a recovery phase following drug application. In separate experiments, we applied either the nAChR agonist nicotine $(n=76)$, or the mAChR agonist oxotremorine $(n=63)$ using micro-iontophoresis.

\section{Cholinergic effects on the contrast response function (CRF)}

We first examine how drug application affected neural responses to gratings of different contrasts averaged across all orientations. The peri-stimulus time histograms and raster plots for neural activity under nicotine application recorded in the granular layer of the primary visual cortex are shown in Fig. 2A-C. As expected, neural activity generally increased with increasing contrast. During nAChR stimulation, neural activity was substantially elevated for this example unit relative to the baseline and recovery phases. To quantify these effects, we performed a two-way ANOVA on the stimulus-evoked neural activity with factors of contrast and experimental phase (baseline vs drug application). For the example recording site, we observed main effects of contrast and experimental phase $(P<0.001$, post hoc tests). Neural activity during baseline and recovery did not differ significantly (two-way ANOVA: $P>0.1$ for main effects and interaction), confirming the stability of the recording. To obtain a single number describing the drug impact on contrast sensitivity, we first plotted the mean firing rate as a function of contrast averaged across stimulus repetitions and then computed the area under the resulting CRF for each experimental phase, as shown in Fig. 2D. For this example unit, nAChR stimulation thus resulted in a robust CRF area increase of $\triangle \mathrm{CRFA}=89$. We fitted Naka-Rushton functions to the contrast response rates, in order to determine whether nAChR stimulation leads to changes in response gain or sensitivity. The data were well fit by these functions for this example recording site, allowing the extraction of peak firing rate $\left(R_{\max }\right)$, semi-saturation contrast $\left(C_{50}\right)$ and spontaneous activity $\left(R_{0}\right)$. How these parameters change with $\mathrm{nAChR}$ and $\mathrm{mAChR}$ agonist application is further analysed below (see Fig. 4). 

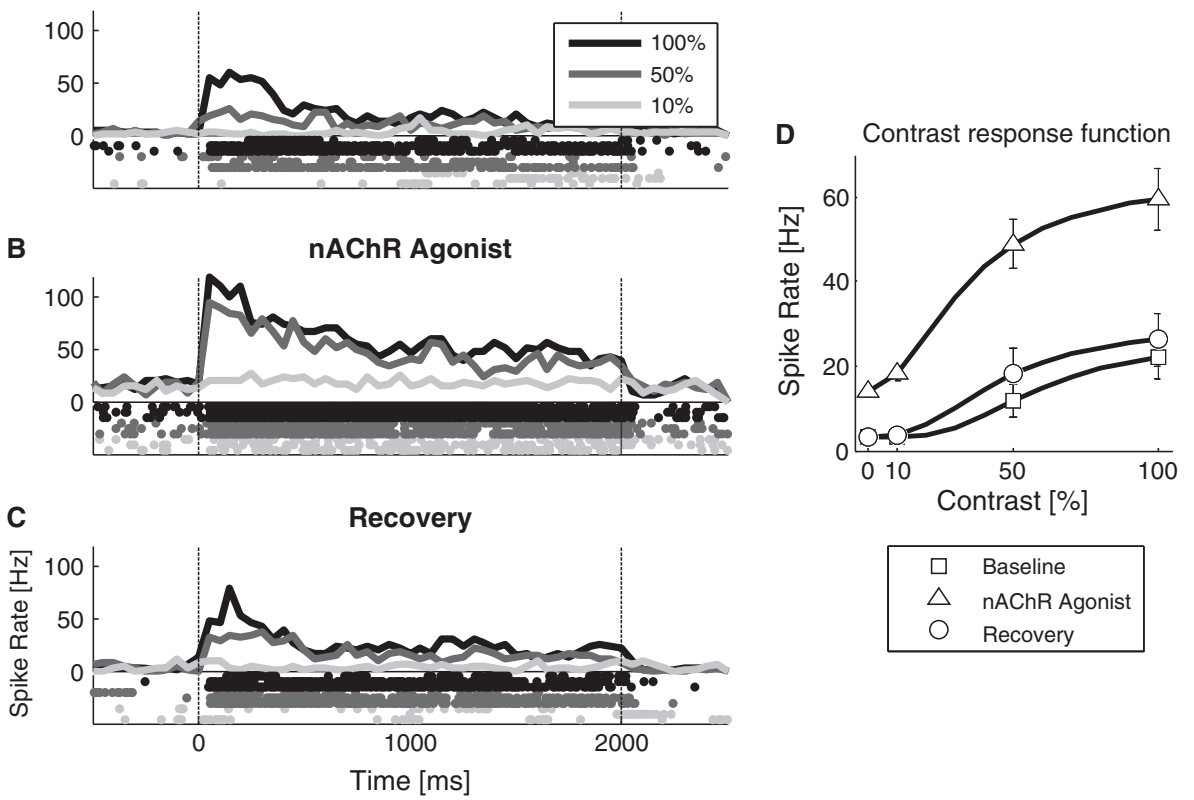

FIG. 2. Single experiment example of increased contrast sensitivity induced by nicotinic acetylcholine receptor (nAChR) stimulation. (A-C) Peri-stimulus time histograms and spike raster plots for $10 \%$ (light gray), 50\% (darker gray) and $100 \%$ contrast (black); note the increased spiking during nicotine application. Three rasters are shown per condition for illustrative purposes. Vertical dashed lines mark on- and offset of the visual stimulus. (D) Contrast response function (CRF) with Naka-Rushton function fits corresponding to baseline, nAChR activation and recovery periods. Error bars denote the SEM.

To examine the effects of nAChR stimulation on the CRF across the population of recorded units, we plotted the value of the CRF area change $\triangle \mathrm{CRFA}$ for each unit against its cortical depth (Fig. 3A). Units, whose activities were significantly modulated by drug application $(n=46)$ according to the two-way ANOVA described above $(P<0.05$ for either main effect of drug or contrast $\times$ drug interaction), are shown as gray circles, the remaining units are shown as black diamonds. The nAChR stimulation had a generally excitatory effect, shown by positive $\triangle$ CRFA values across cortical layers ( $n=42$ units), and there were only very few exceptions $(n=4)$ of units showing negative $\triangle$ CRFA values. The fraction of units $(f)$ affected by nAChR stimulation was greater in the granular layer than the other two layers $\left[f_{\mathrm{sg}}=58 \%(22 / 38)\right.$, $f_{\mathrm{g}}=75 \%(21 / 28), f_{\mathrm{ig}}=30 \%(3 / 10), \chi^{2}$-tests: $\left.P<0.05\right]$, and only a minority of units in the infragranular layer were affected. Directly comparing the magnitude of the $\mathrm{nAChR}$ effect between granular and supragranular layers, we observed higher $\triangle \mathrm{CRFA}$ values in the granular $[(\Delta \mathrm{CRFAg})=49 \mathrm{~Hz}]$ compared with the supragranular $[(\Delta$ CRFAsg $)=15 \mathrm{~Hz}]$ layer (Kruskal-Wallis ANOVA and post hoc tests, $P<0.01$ ). In addition to these effects of nAChR stimulation, we also examined the effects of the mAChR agonist oxotremorine, which was applied during separate experiments. The results, shown in Fig. 3B, indicate that a significantly lower number of units showed a change in CRF area ( $\triangle \mathrm{CRFA}$ ) following the $\mathrm{mAChR}$ stimulation $(25 / 63$ or $40 \%)$ compared to nAChR stimulation $(45 / 76$ or $59 \%, \chi^{2}$-test, $\left.P \ll 0.01\right)$. A similar fraction of units was affected by $\mathrm{mAChR}$ stimulation in all three layers $\left[f_{\mathrm{sg}}=36 \%(14 / 39)\right.$, $f_{\mathrm{g}}=42 \%(5 / 12), f_{\mathrm{ig}}=50 \%(6 / 12), \chi^{2}$-tests: $P>0.1$, Yates correction]. Note, that the Yates correction is recommended to prevent overestimation of statistical significance for low cell count $(n \leq 5)$ $\chi^{2}$-tests; results remain significant without Yates correction also. Along the same lines, a non-parametric ANOVA (Kruskal-Wallis test) revealed no significant differences in $\triangle \mathrm{CRFA}$ between any of the three layers. It is noticeable though that $\mathrm{mAChR}$ stimulation tended to lead almost exclusively to enhancement of the CRFA, particularly in the supragranular layer where not a single recording site showed significant CRFA reduction following drug application. The effects of nAChR and mAChR are summarized in Fig. 3C, which shows the average $\triangle \mathrm{CRFA}$ value for each receptor type and each cortical layer. As can be seen, the effects of nAChR on the CRF of visual cortical neurons are highly layer dependent, with the strongest effects observed in the granular input layer of the cortex. Both mAChR and nAChR activation modestly enhances the contrast response in the supra- and infragranular layers. Overall, mAChR stimulation impacts fewer neurons, has a comparatively weaker effect on the CRF, and exhibits little layer specificity.

We proceeded to fit Naka-Rushton functions (see Materials and methods) to neural CRF recorded during nAChR and mAChR activation (for example, see Fig. 2D above), to analyse whether the observed changes in contrast response are due to shifts in spontaneous activity $R_{0}$, peak firing rate $R_{\max }$ or contrast sensitivity $C_{50}$. As shown in Fig. 4A, the peak response $\left(R_{\max }\right)$ was enhanced weakly for mAChR (baseline: $24.6 \mathrm{~Hz}$; drug: $30.7 \mathrm{~Hz}$, paired $t$-test: $P<0.01$ ) and strongly for nAChR (baseline: $24.7 \mathrm{~Hz}$; drug: $35.9 \mathrm{~Hz}$, paired $t$ test: $P \ll 0.001)$ stimulation, consistent with an enhancement in response gain following cholinergic agonists. On the other hand, contrast sensitivity $\left(C_{50}\right)$ was not systematically affected by either $\operatorname{mAChR}\left(C_{50}\right.$ baseline $=49 \%, C_{50}{ }^{\mathrm{mAChR}}=47 \%$, paired $t$-test: $\left.P>0.1\right)$ or nAChR $\left(C_{50}{ }^{\text {baseline }}=51 \%, C_{50}{ }^{\mathrm{nAChR}}=49 \%\right.$, paired $t$-test: $\left.P>0.1\right)$ application (Fig. 4B). We observed that spontaneous activity was enhanced by mAChR stimulation (baseline: $2.6 \mathrm{~Hz}$; drug: $5.0 \mathrm{~Hz}$, paired $t$-test: $P=0.011$ ) as well as by nAChR stimulation (baseline: $2.6 \mathrm{~Hz}$; drug: $6.3 \mathrm{~Hz}$, paired $t$-test: $P \ll 0.001$ ), as shown in Fig. 4C. Finally, we examined the effect of cholinergic agonists on visually evoked activity $\left(R_{\max }-R_{0}\right)$, to ask whether the peak firing rate was in fact enhanced if the enhanced spontaneous activity $\left(R_{0}\right)$ is taken into account. The results, shown in Fig. 4D, indicate that nAChR stimulation resulted in significant enhancement of visually evoked 

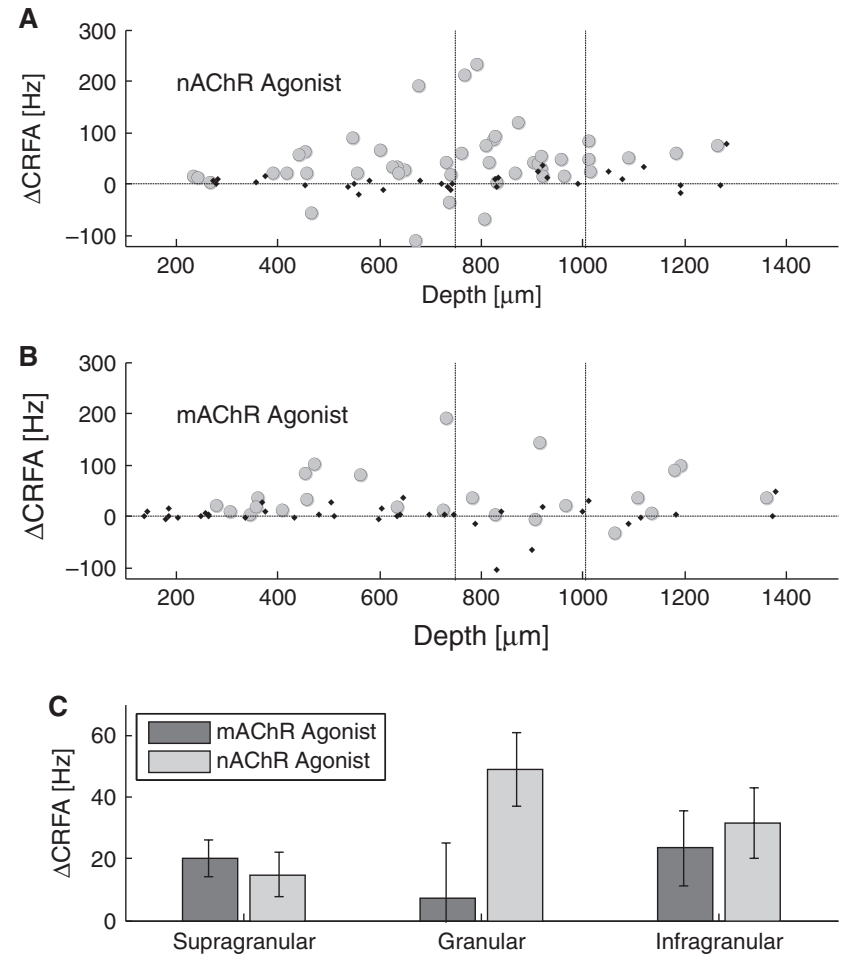

FIG. 3. Population analysis of cholinergic impact on contrast sensitivity. (A) Change in contrast response function area $(\triangle \mathrm{CRFA})$ during nicotinic acetylcholine receptor (nAChR) stimulation plotted against cortical depth, vertical lines denote anatomical borders between supragranular, granular and infragranular layers. A robust increase in CRFA is observed in the granular layer. Units with significant $\triangle \mathrm{CRFA}$ values are shown as gray circles, remaining units as black diamonds. (B) $\triangle \mathrm{CRFA}$ during muscarinic acetylcholine receptor (mAChR) stimulation plotted against cortical depth. Fewer units were affected by $m A C h R$ stimulation and effects were not layer specific. (C) Summary figure showing mean \pm SEM for significantly affected units in $\mathrm{nAChR}$ and $\mathrm{mAChR}$ stimulation conditions.

activity (paired $t$-test: $P<0.01$ ), whereas mAChR stimulation did not (paired $t$-test: $P=0.08$ ).

\section{Cholinergic effects on orientation tuning}

We proceeded to examine whether, in addition to the changes in the neural contrast response described above, layer-specific cholinergic drug application also had an impact on the orientation tuning of V1 units. The peri-stimulus time histograms and raster plots for a single recording site during $\mathrm{mAChR}$ stimulation recorded in the supragranular layer of the primary visual cortex are shown in Fig. 5A-C. During drug application, elevated activity was observed for the preferred orientation, whereas activity for the orthogonal orientation remained similar to levels during the baseline period. During the recovery period after drug application, orientation selectivity of this unit returned to baseline levels. To quantify orientation selectivity, we used an OTI (see Materials and methods) that takes a value of zero for untuned activity and a value of one if responses are seen for only one orientation. The neural activity during the baseline, drug application and recovery epochs are shown in Fig. 5D-F. Because we were interested in orientation and not direction selectivity, we averaged data from direction pairs corresponding to the same orientation (see Materials and methods). As can be seen, the preferred orientation $\left(135^{\circ}\right)$ remained approximately the same during the three epochs, but the OTI value was enhanced during $\mathrm{mAChR}$ stimulation relative to levels during the baseline period. We fitted wrapped normal functions to the orientation response data (see Materials and methods), yielding parameters of TW and TH. An enhanced TH ( $100 \%$ increase) and slightly reduced TW ( $\sim 10 \%$ decrease $)$ values are observed during mAChR stimulation relative to control periods for this example recording. How these parameters change across the population of recorded sites is further analysed below (see Fig. 7).

The distribution of the OTI across layers is shown in Fig. 6A. Orientation tuning was on average lower in the granular input layer of the cortex than in each of the other layers $\left(\mathrm{OTI}_{\mathrm{sg}}=0.22 \pm 0.02\right.$, $\mathrm{OTI}_{\mathrm{g}}=0.12 \pm 0.02, \quad \mathrm{OTI}_{\mathrm{ig}}=0.21 \pm 0.02$, Kruskal-Wallis ANOVA and post hoc tests, $P<0.05$ ). It is indeed clearly visible that strongly orientation tuned units are largely absent from the granular input layer. To examine the effects of drug application across the population, we examined how the OTI values changed between drug and baseline conditions. Because no clear differences were observed regarding cortical layers, these OTI changes are summarized for all layers together in Fig. 6B,C. Effects were generally variable, and orientation tuning could be both enhanced and attenuated by drug application. We did find that during mAChR stimulation, significant increases in OTI occurred significantly more frequently than decreases (28 vs. $16, \chi^{2}$ test, $P<0.01)$. By contrast, nAChR stimulation tended to have the opposite effect: during $\mathrm{nAChR}$ agonist application, significantly more units exhibited reduced OTI values compared with baseline conditions before drug injection ( 35 vs. $21, \chi^{2}$-test, $P<0.01$ ). Note, however, that both mAChR-related enhancement and nAChR-related attenuation of orientation tuning was generally small in magnitude $(+10 \%$ and $-15 \%$, respectively).

To gain further insight into the nature of the changes in orientation tuning, we fitted wrapped normal functions to the orientation response data (see Materials and methods; Fig. 5D-F for examples, yielding the parameters TH and TW), using only sites that were orientation-tuned during the baseline period $\left(\mathrm{OTI}_{\text {baseline }}>0.1\right)$. In Fig. $7 \mathrm{~A}$, we show that TH (i.e. the differences in spiking rate between preferred and nonpreferred orientations) was greater during application of both $\mathrm{mAChR}$

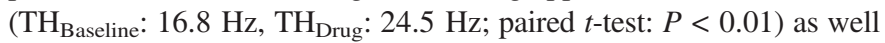
as nAChR $\left(\mathrm{TH}_{\text {Baseline: }} 17.9 \mathrm{~Hz}, \mathrm{TH}_{\text {Drug: }}: 24.3 \mathrm{~Hz}\right.$; paired $t$-test: $P<0.001)$. As shown in Fig. 7B, TW was unaffected by mAChR stimulation $\left.\left(\mathrm{TW}_{\text {Baseline }}=90^{\circ}, \mathrm{TW}_{\text {Drug }}=91^{\circ}\right) ; P>0.1\right)$, but significantly increased following nAChR stimulation $\left(\mathrm{TW}_{\text {Baseline }}=84^{\circ}\right.$, $\left.\mathrm{TW}_{\text {Drug }}=98^{\circ} ; P<0.001\right)$. Together, these findings indicate that both $\mathrm{mAChR}$ and $\mathrm{nAChR}$ stimulation enhance TH, that is they lead to an enhancement in differential activity to preferred and non-preferred orientation stimuli. However, while TW increases for nAChR stimulation, it remains stable for mAChR stimulation. These findings parallel observations made using the OTI, which suggest slight enhancement and reduction of orientation tuning following $\mathrm{mAChR}$ and nAChR stimulation, respectively.

\section{Recording stability analysis}

During drug application studies, it is important to ensure that putative drug effects are actually caused by drug application and not by nonspecific fluctuations in neural activity, for example due to instability of recordings. To address this issue, we performed a two-way ANOVA with contrast and baseline/recovery as factors on neural activity during $\mathrm{nAChR}$ or $\mathrm{mAChR}$ experiments. This analysis revealed that, of the recordings with available recovery epoch activity (133/139), about half $(66 / 133$ or $50 \%)$ showed no difference in neural activity between baseline and recovery periods $(P>0.1$ for main effect of 

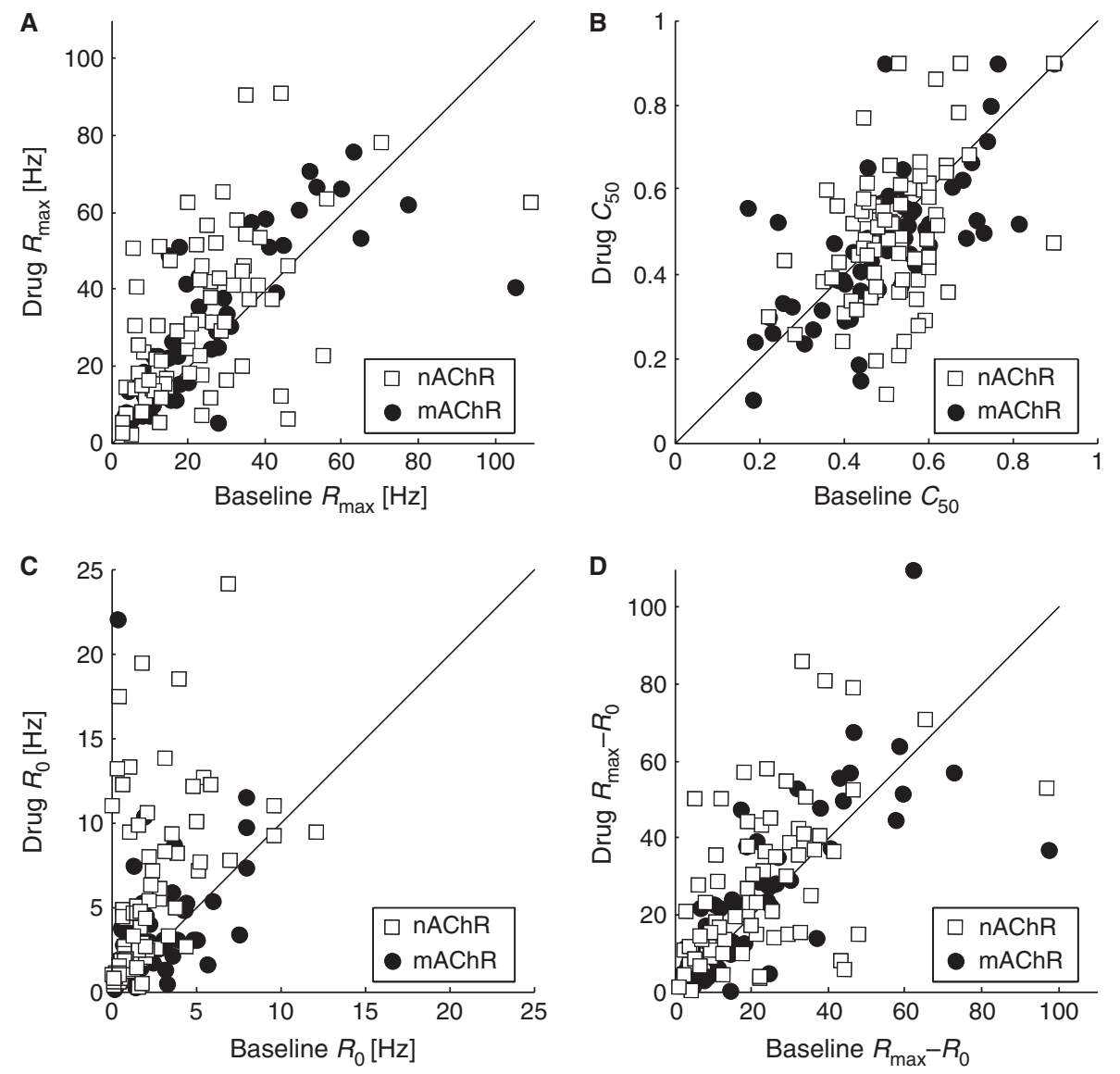

FIG. 4. Population data for Naka-Rushton fit parameters during nicotinic acetylcholine receptor (nAChR) and muscarinic acetylcholine receptor (mAChR) stimulation. (A) Peak firing rate $R_{\max }$. (B) Contrast sensitivity $C_{50}$. (C) Spontaneous activity $R_{0}$. $R_{\max }$ and $R_{0}$ were enhanced, and $C_{50}$ was unaffected for both nAChR and mAChR application. (D) Visually evoked activity $\left(R_{\max }-R_{0}\right)$ was enhanced only during nAChR application.

epoch and contrast $\times$ epoch interaction). At these sites, activity had thus fully recovered from the drug application to baseline levels of contrast sensitivity. The remaining units $(67 / 133)$ did not exhibit full recovery to baseline levels, probably due to lingering effects of drug application on V1 network activity during the recovery epoch, which was recorded immediately after drug application. However, $\triangle$ CRFA did significantly decrease from the drug application to the recovery period (non-parametric ANOVA or Kruskal-Wallis test, $P<0.001$ ). Taken together, these analyses suggest that effects observed during the drug application period were related to drug application, rather than resulting from non-specific fluctuations in neural activity. We compared the two populations of fully recovering and partially recovering units in terms of the major analyses reported above, and found very similar results for these two populations of units compared with the analyses reported above.

\section{Discussion}

We examined the influence of nicotinic and muscarinic activation of the cholinergic system on information processing in tree shrew primary visual cortex. Comparing contrast response and orientation selectivity across layers, we found a dissociation of cholinergic effects with respect to laminar position and receptor type. Nicotinic activation led to a robust increase in response gain restricted largely to the thalamo-recipient granular layer, while weakly reducing orientation tuning across all layers. By contrast, muscarinic activation exhibited little layer specificity, modestly enhancing contrast gain while tending to improve orientation tuning.

We recorded neural activity with laminar resolution in the primary visual cortex, under the application of $\mathrm{mAChR}$ and $\mathrm{nAChR}$ agonists and by varying not only the contrast, but also the orientation of grating stimuli presented in the receptive field of the unit under investigation. Pharmacological agents were delivered iontophoretically in the close vicinity of the unit under study using an electrode-pipette combination. A major advantage of layer-specific recordings is that findings can be examined in the context of canonical microcircuit models of the cortex (Hirsch \& Martinez, 2006a; Binzegger et al., 2009). These models provide a comprehensive account about connectivity, information flow and division of labor among the layers of primary visual cortex, and thus provide a useful framework into which the cholinergic neuromodulation effects can be integrated, if the laminar position of recorded neural activity is available. Current efforts for understanding cholinergic effects on brain function are pursuing similar goals, for example in the auditory cortex (Metherate, 2010) and the olfactory bulb (Linster \& Cleland, 2002; Mandairon et al., 2006), by focusing on building models that integrate functional data with anatomical information. In Fig. 8, we present a simplified diagram of a cortical microcircuit illustrating the layer-specific cholinergic receptor densities. We combined cortical connectivity data (Binzegger et al., 2009) with cholinergic receptor distribution data (Disney et al., 2006, 2007), while omitting connections that are not relevant for the discussion below. The sensory information from the retina is carried by thalamic 
A

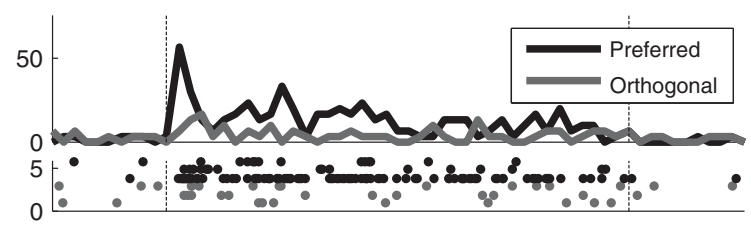

B

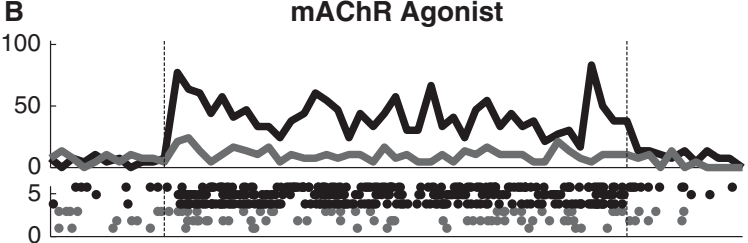

D $\mathrm{TW}: 89^{\circ} \mathrm{TH}: 9 \mathrm{~Hz}$

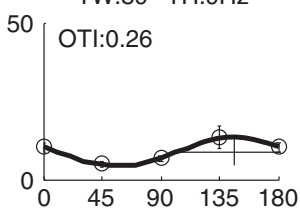

E

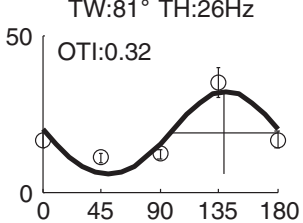

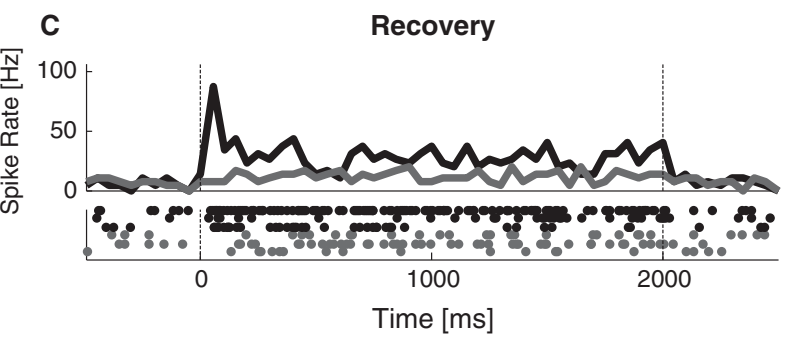

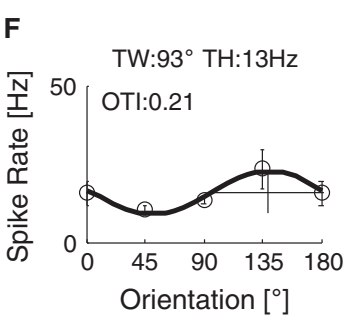

FIG. 5. Single experiment example of increased orientation tuning following muscarinic acetylcholine receptor (mAChR) stimulation. (A-C) Peri-stimulus time histograms and spike raster plots for preferred orientation (black) and orthogonal orientation (light gray). Vertical dotted lines denote on- and offset of the visual stimulus. Note the difference in activity between preferred and orthogonal orientation during drug application. (D-F) Orientation tuning corresponding to (A-C) showing mean spike rate \pm SEM and the wrapped Gaussian fits (solid lines). Values of the orientation tuning index (OTI), and the tuning width (TW) and tuning height $(\mathrm{TH})$ parameters of wrapped Gaussian fits to the data are also shown.
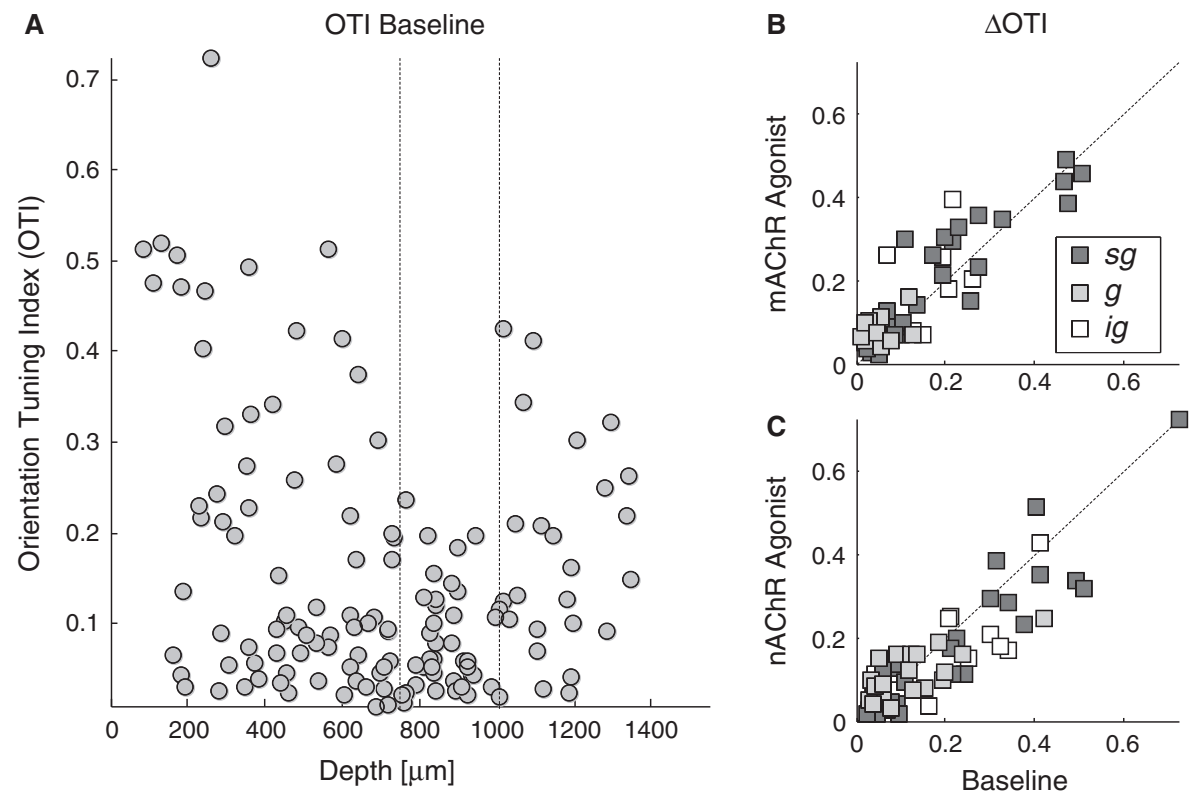

FIG. 6. Population analysis of cholinergic impact on orientation tuning. (A) Orientation tuning indices (OTIs) for all units recorded in the baseline condition prior to drug application. Units in the supragranular and infragranular layers show stronger orientation tuning than those in the granular layer. (B) Effect of muscarinic acetylcholine receptor (mAChR) stimulation on the OTI. (C) Effect of nicotinic acetylcholine receptor (nAChR) stimulation on the OTI. Sg, g and ig denote supragranular, granular and infragranular layers, respectively.

axons that synapse on spiny stellate cells in the granular layer of the primary visual cortex. There is a high density of nicotinic receptors present on their axon terminals (Prusky et al., 1987; Sahin et al.,
1992; Disney et al., 2007), and their activation is thought to underlie the response gain increase of thalamic signals observed in our study as well as previous in vivo studies in the visual (Disney et al., 2007; 

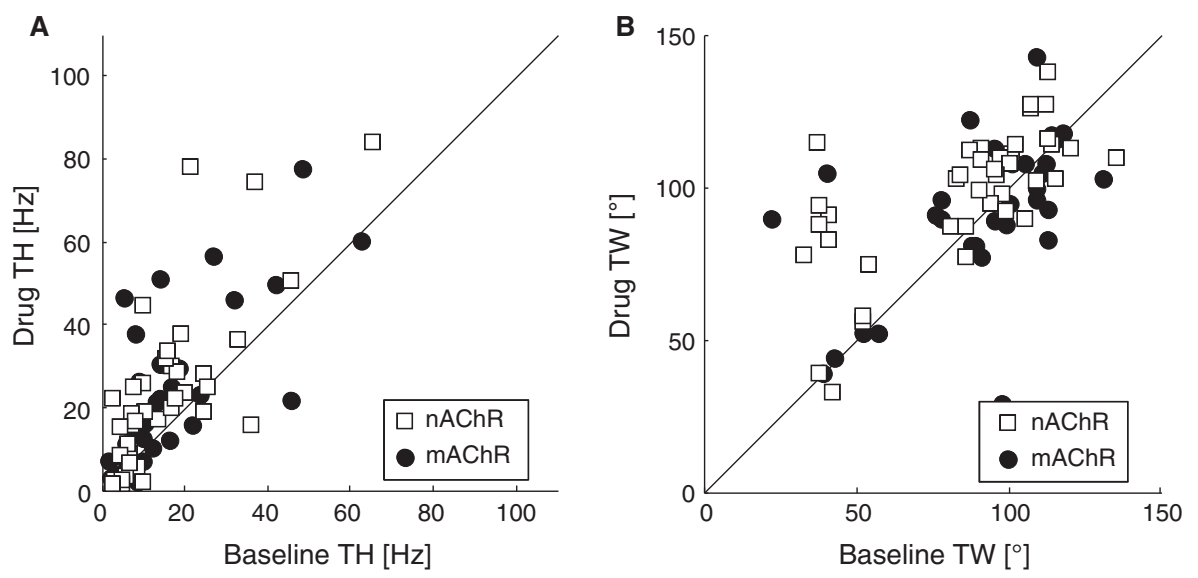

FIG. 7. Population data for wrapped Gaussian fits to orientation tuning data for nicotinic acetylcholine receptor (nAChR) and muscarinic acetylcholine receptor (mAChR) stimulation. (A) Tuning height (TH). (B) Tuning width (TW). Both nAChR and mAChR stimulation increased TH, whereas TW was increased for nAChR but stable for mAChR stimulation.
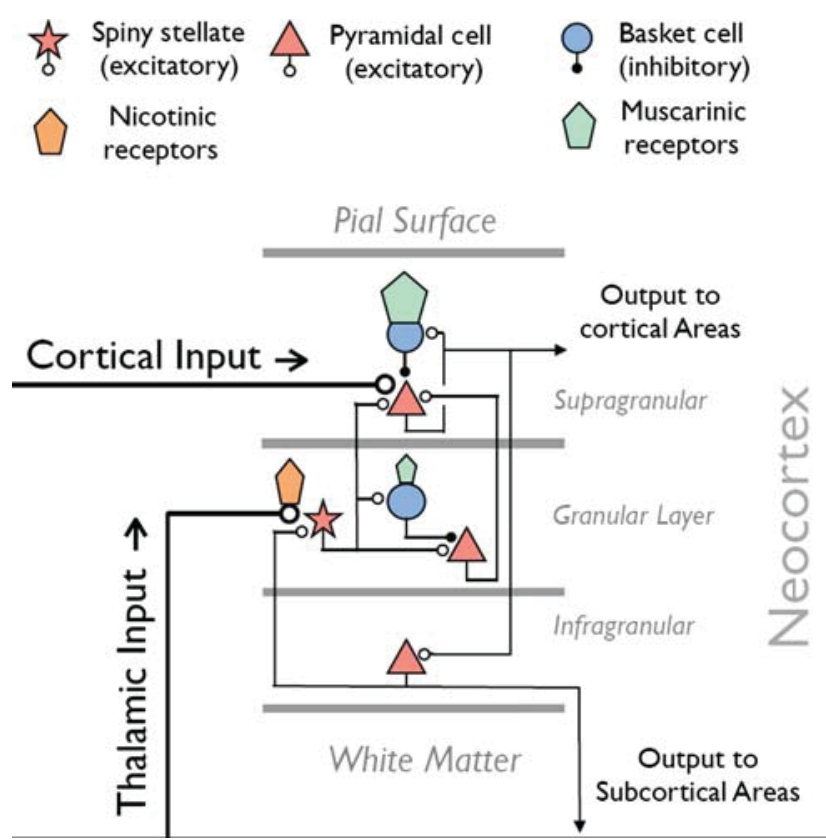

Fig. 8. Simplified diagram of the laminar cortical microcircuit. Excitatory cells are red (synapse indicated by empty circle), inhibitory cells blue (synapse indicated by filled circle); spiny stellate cells in the granular layer receive thalamic input; activation of nAChRs (orange) on the thalamo-cortical axons can amplify sensory input. Neurons in the supragranular layers receive cortical feedback signals and integrate information from granular layer. mAChRs (green) are expressed on inhibitory interneurons in all layers, the highest density is found in supragranular layers. Activation of mAChRs leads to inhibition of (local and global) cortical feedback, and this suppression of intracortical signals contributes to enhanced orientation selectivity.

Soma et al., 2011) and somatosensory (Oldford \& Castro-Alamancos, 2003) cortex. Although spontaneous activity in the absence of visual stimulation was also elevated by nAChR activation in our study, the enhancement in visual-evoked activity was highly significant after subtraction of this spontaneous activity. In addition to the increased gain, we have found that $\mathrm{nAChR}$ agonist application resulted in a reduction of orientation selectivity in all cortical layers. This may result from a response gain increase associated with nicotine, which generally boosts neural activity and may thus dampen differential activations due to orientation differences. This is consistent with the increased TW of the orientation tuning function we observed for $\mathrm{nAChR}$ activation. Another possibility is that reduced orientation selectivity may result from activation of nAChRs present on inhibitory interneurons (Aracri et al., 2010), which regulate $\gamma$-aminobutyric acid (GABA) release and thereby affect neural selectivity to orientation. As discussed below, inhibitory networks play an important role in shaping orientation selectivity in the cortex, and nAChR activation may interfere with these computations.

During mAChR activation using oxotremorine, we observed a non layer-specific enhancement of the contrast response, similar to a recent study in macaque V1 (Soma et al., 2011), but reduced in terms of effect size. Although we cannot rule out species differences as contributing to this result, we suggest that it might in fact be due to differences in receptor-binding affinity between oxotremorine, which we used in our study and which is more specific for M2 than M1 receptors, and atropine, used by Soma and colleagues, which targets preferentially M1 receptors (Peralta et al., 1987). This suggests that the strong effects on response gain observed by Soma and colleagues are mostly due to M1 receptors. Muscarinic receptors have been shown to be most strongly expressed in supragranular layers (Levey et al., 1991; Disney et al., 2006; Eickhoff et al., 2007). Muscarinic activation has been closely linked to intracortical inhibition: early in vitro studies have already suggested that muscarinic effects in the cortex are mediated mostly by activating GABAergic inhibition (McCormick \& Prince, 1986), and more recent work has shown that some pyramidal cells also express mAChRs, and glutamate release can thus be directly inhibited by acetylcholine (Gulledge \& Stuart, 2005; Amar et al., 2010). In addition, inhibitory GABAergic synapses show a similar distribution to $\mathrm{mAChRs}$ with relatively high abundance observed in the supragranular layers (Fitzpatrick et al., 1987; Eickhoff et al., 2007). In the present study, we observed the strongest orientation selectivity outside of the granular layer, in both supraand infragranular layers. Interestingly, cells with low OTI values below 0.1 are almost absent in the infragranular layers, whereas they are abundant in granular and supragranular layers (see Fig. 6). These observations are consistent with the idea that orientation selectivity is generated in the supragranular layer by anatomical asymmetries of feed-forward inputs from the granular layer (Mooser et al., 2004) and refinement of responses by supragranular inhibitory circuits (Tsumoto et al., 1979; Sato et al., 1995; Fitzpatrick, 1996; Hirsch \& Martinez, $2006 a, b)$. The oriented neural responses are then fed forward to the 
infragranular layers (Binzegger et al., 2009), explaining the lower abundance of unoriented cells in these laminae. Following mAChR stimulation, we observed a modest increase in orientation selectivity across cortical layers, consistent with previous evidence that muscarinic activation can enhance stimulus-selective responses (Sillito \& Kemp, 1983; Sato et al., 1987; Murphy \& Sillito, 1991). Our data thus reveal that $\mathrm{mAChR}$ and $\mathrm{nAChR}$ stimulation appear to have opposite effects on orientation tuning of visual cortical neurons, leading to enhancement and suppression of OTIs, respectively. This may explain why administration of acetylcholine did not lead to systematic changes in orientation tuning (Zinke et al., 2006), because acteylcholine stimulates both receptor types and receptor subtype-specific effects may thus have cancelled each other.

Reported effects of nicotine on visual behavior are largely consistent with the robust enhancement in V1 response gain we observed in our study. In rodents, many studies have reported effects of nicotinic receptor agonists on various behavioral parameters in visual detection paradigms, the most consistent observation being a reduction in reaction times (Grilly et al., 2000; Phillips et al., 2000; Hahn et al., 2002). Most closely related to our results, one study has demonstrated that systemic administration of a $\alpha 4 \beta 2 \mathrm{nAChR}$ agonist enhanced stimulus detection and reduced reaction times (Grottick \& Higgins, 2000). These are the behavioral effects that one would predict based on the observation of enhanced response gain due to the activation of $\alpha 4 \beta 2 \mathrm{nAChR}$ on the thalamo-cortical synapses in the V1 granular layer. These results are paralleled by psychophysical findings in humans, where nicotine has also been associated with speeding of reaction times in diverse paradigms (Wesnes \& Warburton, 1984; Warburton \& Mancuso, 1998; Mirza \& Stolerman, 2000; Griesar et al., 2002). An emerging view is that many of the effects associated with nicotine in human studies may in fact result from drug action in higher cortical areas (Lawrence et al., 2002; Meinke et al., 2006; Hahn et al., 2009). However, a specific elevation of the contrast threshold for the detection of grating stimuli following nicotine application has been demonstrated (Smith \& Baker-Short, 1993), which is likely to result from nicotine action on the $\alpha 4 \beta 2$ nAChR on the thalamo-cortical synapses. Also consistent with this, a positron emission tomography study has demonstrated increased blood flow in the visual thalamus during a visual task following cholinergic stimulation, in a manner consistent with nAChR activation (Mentis et al., 2001).

Muscarinic effects on behavior have also been extensively characterized, with investigations often focusing on disruption of $\mathrm{mAChR}$ transmission using the receptor antagonist scopolamine (Klinkenberg \& Blokland, 2010). A selective behavioral impairment following mAChR blockade has previously been demonstrated in a forced swimming paradigm in rats and mice (Robinson et al., 2004), where application of the mAChR antagonist scopolamine resulted in impaired visual acuity, such that animals were less able to discriminate between visual gratings of different spatial frequencies. Spatial frequency selectivity, like orientation selectivity, is elaborated in the cortex (Shapley \& Lennie, 1985), and our findings suggest that interference with mACh receptors in $\mathrm{V} 1$ may underlie the behavioral deficits observed in this study. Another study has demonstrated that an immunotoxic lesion of the cholinergic system impairs learning of fine orientation discrimination in rats, suggesting a cholinergic role in shaping neural circuits in V1 during perceptual discrimination learning (Dotigny et al., 2008). Although an immunotoxic lesion affects both nicotinic and muscarinic neurotransmission, our results suggest that it is effects of acetylcholine on $\mathrm{V} 1 \mathrm{mACh}$ receptors that underlie the observed improvement of behavioral orientation discrimination. This idea is supported by findings indicating that prolonged pairing of visual stimulation with $\mathrm{mAChR}$ activation strongly potentiates visual- evoked potentials in rat visual cortex, by activating long-term potentiation involving the $N$-methyl-D-aspartate glutamate receptor (Kang \& Vaucher, 2009). Finally, muscarinic activation has been shown to enhance attention-related increases in neural activity in the macaque primary visual cortex (Herrero et al., 2008). Attention is traditionally regarded as a top-down mechanism, by which higher brain areas steer information flow through sensory cortices, and attentional effects would thus be expected to occur in supragranular layers of $\mathrm{V} 1$, which process cortico-cortical communication.

Our findings are generally consistent with models of cholinergic function (Hasselmo, 2006), which conceptualize the role of acetylcholine as balancing the relative contribution of bottom-up input and top-down feedback in the cortex. High levels of acetylcholine are associated with enhanced bottom-up and suppressed top-down signals. The increase in bottom-up input is assumed to be mediated via nicotinic activation, whereas the suppression of top-down feedback is thought to depend on muscarinic receptors. Our study provides confirmation of these ideas in the primary visual cortex. By taking cortical layer and receptor type into account, we can indeed associate an increase in bottom-up signal gain with nicotinic activation in the granular layer, and inhibition of top-down recurrent feedback with muscarinic action predominant in the supragranular layer. The designation of specific computational functions to cholinergic neuromodulation may seem at odds with the diffuse nature of cholinergic projections to the cortex from the basal forebrain (Mesulam et al., 1992). However, our results further reinforce the idea that due to differences in receptor distribution between cortical layers and neuron types, acetylcholine can have spatially precise effects on sensory representations. This spatial specificity is complemented by high temporal precision (Sarter et al., 2009), and thus allows cholinergic neuromodulation to play an important role in sensory coding at timescales relevant for perception. Taken together, our results reveal that the cortical layer in which neural activity is recorded plays a major role in determining the effects induced by stimulation of cholinergic receptors. Our data, obtained in the tree shrew, fit well with available data from other species, including findings in the macaque monkey (Disney et al., 2007; Soma et al., 2011). We have recently reported striking similarities between macaque and tree shrew V1 in terms of temporal precision of neural responses (Veit et al., 2011). The present findings extend the documented homologies between these species to the domain of cholinergic neuromodulation, further highlighting the usefulness of the tree shrew as an animal model for studying visual processing.

\section{Acknowledgements}

This work was supported by SNF Prodoc grant PDFMP3 127179, the University of Fribourg and a European Young Investigator Award to G.R.

\section{Abbreviations}

CRF, contrast response function; GABA, $\gamma$-aminobutyric acid; mAChR, muscarinic acetylcholine receptor; nAChR, nicotinic acetylcholine receptor; OTI, orientation tuning index; TH, tuning height; TW, tuning width.

\section{References}

Amar, M., Lucas-Meunier, E., Baux, G. \& Fossier, P. (2010) Blockade of different muscarinic receptor subtypes changes the equilibrium between excitation and inhibition in rat visual cortex. Neuroscience, 169, 1610-1620. Aracri, P., Consonni, S., Morini, R., Perrella, M., Rodighiero, S., Amadeo, A. \& Becchetti, A. (2010) Tonic modulation of GABA release by nicotinic 
acetylcholine receptors in layer $\mathrm{V}$ of the murine prefrontal cortex. Cereb. Cortex, 20, 1539-1555.

Binzegger, T., Douglas, R.J. \& Martin, K.A. (2009) Topology and dynamics of the canonical circuit of cat V1. Neural Netw., 22, 1071-1078.

Brown, D.A. (2010) Muscarinic acetylcholine receptors (mAChRs) in the nervous system: some functions and mechanisms. J. Mol. Neurosci., 41 340-346.

Chapman, B. \& Stryker, M.P. (1992) Origin of orientation tuning in the visual cortex. Curr. Opin. Neurobiol., 2, 498-501.

Dani, J.A. \& Bertrand, D. (2007) Nicotinic acetylcholine receptors and nicotinic cholinergic mechanisms of the central nervous system. Annu. Rev. Pharmacol. Toxicol., 47, 699-729.

Disney, A.A., Domakonda, K.V. \& Aoki, C. (2006) Differential expression of muscarinic acetylcholine receptors across excitatory and inhibitory cells in visual cortical areas V1 and V2 of the macaque monkey. J. Comp. Neurol., 499, 49-63.

Disney, A.A., Aoki, C. \& Hawken, M.J. (2007) Gain modulation by nicotine in macaque v1. Neuron, 56, 701-713.

Dotigny, F., Ben Amor, A.Y., Burke, M. \& Vaucher, E. (2008) Neuromodulatory role of acetylcholine in visually-induced cortical activation: behavioral and neuroanatomical correlates. Neuroscience, 154 1607-1618.

Eickhoff, S.B., Rottschy, C. \& Zilles, K. (2007) Laminar distribution and codistribution of neurotransmitter receptors in early human visual cortex. Brain Struct. Funct., 212, 255-267.

Fitzpatrick, D. (1996) The functional organization of local circuits in visual cortex: insights from the study of tree shrew striate cortex. Cereb. Cortex, $\mathbf{6}$ 329-341.

Fitzpatrick, D., Lund, J.S., Schmechel, D.E. \& Towles, A.C. (1987) Distribution of GABAergic neurons and axon terminals in the macaque striate cortex. J. Comp. Neurol., 264, 73-91.

Fournier, G.N., Semba, K. \& Rasmusson, D.D. (2004) Modality- and regionspecific acetylcholine release in the rat neocortex. Neuroscience, 126, 257262.

Griesar, W.S., Zajdel, D.P. \& Oken, B.S. (2002) Nicotine effects on alertnes and spatial attention in non-smokers. Nicotine Tob. Res., 4, 185-194.

Grilly, D.M., Simon, B.B. \& Levin, E.D. (2000) Nicotine enhances stimulus detection performance of middle- and old-aged rats: a longitudinal study. Pharmacol. Biochem. Behav., 65, 665-670.

Grottick, A.J. \& Higgins, G.A. (2000) Effect of subtype selective nicotinic compounds on attention as assessed by the five-choice serial reaction time task. Behav. Brain Res., 117, 197-208.

Gulledge, A.T. \& Stuart, G.J. (2005) Cholinergic inhibition of neocortical pyramidal neurons. J. Neurosci., 25, 10308-10320.

Hahn, B., Shoaib, M. \& Stolerman, I.P. (2002) Nicotine-induced enhancement of attention in the five-choice serial reaction time task: the influence of task demands. Psychopharmacology, 162, 129-137.

Hahn, B., Ross, T.J., Wolkenberg, F.A., Shakleya, D.M., Huestis, M.A. \& Stein, E.A. (2009) Performance effects of nicotine during selective attention, divided attention, and simple stimulus detection: an fMRI study. Cereb. Cortex, 19, 1990-2000.

Hasselmo, M.E. (2006) The role of acetylcholine in learning and memory. Curr. Opin. Neurobiol., 16, 710-715.

Hasselmo, M.E. \& Sarter, M. (2011) Modes and models of forebrain cholinergic neuromodulation of cognition. Neuropsychopharmacology, $\mathbf{3 6}$, $52-73$.

Herrero, J.L., Roberts, M.J., Delicato, L.S., Gieselmann, M.A., Dayan, P. \& Thiele, A. (2008) Acetylcholine contributes through muscarinic receptors to attentional modulation in V1. Nature, 454, 1110-1114.

Hirsch, J.A. \& Martinez, L.M. (2006a) Circuits that build visual cortical receptive fields. Trends Neurosci., 29, 30-39.

Hirsch, J.A. \& Martinez, L.M. (2006b) Laminar processing in the visual cortical column. Curr. Opin. Neurobiol., 16, 377-384.

Kang, J.I. \& Vaucher, E. (2009) Cholinergic pairing with visual activation results in long-term enhancement of visual evoked potentials. PLoS One, 4, e5995.

Klinkenberg, I. \& Blokland, A. (2010) The validity of scopolamine as a pharmacological model for cognitive impairment: a review of animal behavioral studies. Neurosci. Biobehav. Rev., 34, 1307-1350.

Laplante, F., Morin, Y., Quirion, R. \& Vaucher, E. (2005) Acetylcholine release is elicited in the visual cortex, but not in the prefrontal cortex, by patterned visual stimulation: a dual in vivo microdialysis study with functional correlates in the rat brain. Neuroscience, 132, 501-510.

Lawrence, N.S., Ross, T.J. \& Stein, E.A. (2002) Cognitive mechanisms of nicotine on visual attention. Neuron, 36, 539-548.
Levey, A.I., Kitt, C.A., Simonds, W.F., Price, D.L. \& Brann, M.R. (1991) Identification and localization of muscarinic acetylcholine receptor proteins in brain with subtype-specific antibodies. J. Neurosci., 11, 3218-3226.

Linster, C. \& Cleland, T.A. (2002) Cholinergic modulation of sensory representations in the olfactory bulb. Neural Netw., 15, 709-717.

Lucas-Meunier, E., Fossier, P., Baux, G. \& Amar, M. (2003) Cholinergic modulation of the cortical neuronal network. Pflugers Arch., 446, 17-29.

Mandairon, N., Ferretti, C.J., Stack, C.M., Rubin, D.B., Cleland, T.A. \& Linster, C. (2006) Cholinergic modulation in the olfactory bulb influences spontaneous olfactory discrimination in adult rats. Eur. J. Neurosci., 24, 3234-3244.

McCormick, D.A. \& Prince, D.A. (1986) Mechanisms of action of acetylcholine in the guinea-pig cerebral cortex in vitro. J. Physiol., 375 , 169-194.

Meinke, A., Thiel, C.M. \& Fink, G.R. (2006) Effects of nicotine on visuospatial selective attention as indexed by event-related potentials. Neuroscience, 141, 201-212.

Mentis, M.J., Sunderland, T., Lai, J., Connolly, C., Krasuski, J., Levine, B., Friz, J., Sobti, S., Schapiro, M. \& Rapoport, S.I. (2001) Muscarinic versus nicotinic modulation of a visual task. a pet study using drug probes. Neuropsychopharmacology, 25, 555-564.

Mesulam, M.M., Hersh, L.B., Mash, D.C. \& Geula, C. (1992) Differential cholinergic innervation within functional subdivisions of the human cerebral cortex: a choline acetyltransferase study. J. Comp. Neurol., 318, 316-328.

Metherate, R. (2011) Functional connectivity and cholinergic modulation in auditory cortex. Neurosci. Biobehav. Rev., 35, 2058-2063.

Mirza, N.R. \& Stolerman, I.P. (2000) The role of nicotinic and muscarinic acetylcholine receptors in attention. Psychopharmacology, 148, 243 250.

Mooser, F., Bosking, W.H. \& Fitzpatrick, D. (2004) A morphological basis for orientation tuning in primary visual cortex. Nat. Neurosci., 7, 872879

Murphy, P.C. \& Sillito, A.M. (1991) Cholinergic enhancement of direction selectivity in the visual cortex of the cat. Neuroscience, 40, 13-20.

Oldford, E. \& Castro-Alamancos, M.A. (2003) Input-specific effects of acetylcholine on sensory and intracortical evoked responses in the "barrel cortex" in vivo. Neuroscience, 117, 769-778.

Peralta, E.G., Ashkenazi, A., Winslow, J.W., Smith, D.H., Ramachandran, J. \& Capon, D.J. (1987) Distinct primary structures, ligand-binding properties and tissue-specific expression of four human muscarinic acetylcholine receptors. EMBO J., 6, 3923-3929.

Phillips, J.M., McAlonan, K., Robb, W.G. \& Brown, V.J. (2000) Cholinergic neurotransmission influences covert orientation of visuospatial attention in the rat. Psychopharmacology, 150, 112-116.

Prusky, G.T., Shaw, C. \& Cynader, M.S. (1987) Nicotine receptors are located on lateral geniculate nucleus terminals in cat visual cortex. Brain Res., 412, 131-138.

Reid, R.C. \& Alonso, J.M. (1996) The processing and encoding of information in the visual cortex. Curr. Opin. Neurobiol., 6, 475-480.

Roberts, M.J. \& Thiele, A. (2008) Spatial integration and its moderation by attention and acetylcholine. Front. Biosci., 13, 3742-3759.

Robinson, L., Harbaran, D. \& Riedel, G. (2004) Visual acuity in the water maze: sensitivity to muscarinic receptor blockade in rats and mice. Behav. Brain Res., 151, 277-286.

Sahin, M., Bowen, W.D. \& Donoghue, J.P. (1992) Location of nicotinic and muscarinic cholinergic and mu-opiate receptors in rat cerebral neocortex: evidence from thalamic and cortical lesions. Brain Res., 579, 135-147.

Sarter, M., Parikh, V. \& Howe, W.M. (2009) Phasic acetylcholine release and the volume transmission hypothesis: time to move on. Nat. Rev. Neurosci., 10, 383-390.

Sato, H., Hata, Y., Masui, H. \& Tsumoto, T. (1987) A functional role of cholinergic innervation to neurons in the cat visual cortex. J. Neurophysiol., 58, 765-780.

Sato, H., Katsuyama, N., Tamura, H., Hata, Y. \& Tsumoto, T. (1995) Mechanisms underlying direction selectivity of neurons in the primary visual cortex of the macaque. J. Neurophysiol., 74, 1382-1394.

Shapley, R. \& Lennie, P. (1985) Spatial frequency analysis in the visual system. Annu. Rev. Neurosci., 8, 547-583.

Shapley, R., Hawken, M. \& Ringach, D.L. (2003) Dynamics of orientation selectivity in the primary visual cortex and the importance of cortical inhibition. Neuron, 38, 689-699.

Sillito, A.M. \& Kemp, J.A. (1983) Cholinergic modulation of the functional organization of the cat visual cortex. Brain Res., 289, 143-155. 
Smith, A.T. \& Baker-Short, C.M. (1993) Pharmacological separation of mechanisms contributing to human contrast sensitivity. Vis. Neurosci., 10, 1073-1079.

Soma, S., Shimegi, S., Osaki, H. \& Sato, H. (2012) Cholinergic modulation of response gain in the primary visual cortex of the macaque. J. Neurophysiol., 107, 283-291

Thiele, A., Delicato, L.S., Roberts, M.J. \& Gieselmann, M.A. (2006) A novel electrode-pipette design for simultaneous recording of extracellular spikes and iontophoretic drug application in awake behaving monkeys. J. Neurosci. Methods, 158, 207-211.

Tsumoto, T., Eckart, W. \& Creutzfeldt, O.D. (1979) Modification of orientation sensitivity of cat visual cortex neurons by removal of GABA-mediated inhibition. Exp. Brain Res., 34, 351-363.

Veit, J., Bhattacharyya, A., Kretz, R. \& Rainer, G. (2011) Neural response dynamics of spiking and local field potential activity depend on CRT monitor refresh-rate in the tree shrew primary visual cortex. J. Neurophysiol., 106, 2303-2313.

Warburton, D.M. \& Mancuso, G. (1998) Evaluation of the information processing and mood effects of a transdermal nicotine patch. Psychopharmacology, 135, 305-310.

Wesnes, K. \& Warburton, D.M. (1984) Effects of scopolamine and nicotine on human rapid information processing performance. Psychopharmacology, 82, $147-150$.

Wong-Riley, M. (1979) Changes in the visual system of monocularly sutured or enucleated cats demonstrable with cytochrome oxidase histochemistry. Brain Res., 171, 11-28.

Zinke, W., Roberts, M.J., Guo, K., McDonald, J.S., Robertson, R. \& Thiele, A. (2006) Cholinergic modulation of response properties and orientation tuning of neurons in primary visual cortex of anaesthetized Marmoset monkeys. Eur. J. Neurosci., 24, 314-328. 